\title{
Association of plasma retinol levels with incident cancer risk in Chinese hypertensive adults: a nested case-control study
}

\author{
Liling Xie ${ }^{1}$, Yun Song 2 , Tengfei Lin ${ }^{2}$, Huiyuan $\mathrm{GuO}^{2}$, Binyan Wang ${ }^{3}$, Genfu Tang ${ }^{3}$, Chengzhang Liu ${ }^{4}$, \\ Weitong Huang ${ }^{5}$, Yan Yang ${ }^{6,7}$, Wenhua Ling ${ }^{7,8}$, Yan Zhang 9 , Jianping Li ${ }^{9}$, Yong Huo ${ }^{9}$, Xiaobin Wang ${ }^{10}$, \\ Hao Zhang ${ }^{2 *}$, Xianhui Qin ${ }^{1 *}$ and Xiping Xu ${ }^{1,2,3 *}$ \\ ${ }^{1}$ National Clinical Research Center for Kidney Disease, The State Key Laboratory for Organ Failure Research, Renal Division, \\ Nanfang Hospital, Southern Medical University, Guangzhou 510515, People's Republic of China \\ ${ }^{2}$ Beijing Advanced Innovation Center for Food Nutrition and Human Health, College of Food Science and Nutritional \\ Engineering, China Agricultural University, Beijing 100083, People's Republic of China \\ ${ }^{3}$ Institute of Biomedicine, Anhui Medical University, Hefei 230032, People's Republic of China \\ ${ }^{4}$ Shenzhen Evergreen Medical Institute, Shenzhen 518057, People's Republic of China \\ ${ }^{5}$ Nanning Maternal and Child Health Care Hospital, Nanning 530011, People's Republic of China \\ ${ }^{6}$ School of Public Health (Shenzhen), Sun Yat-Sen University, Guangzhou 510006, People's Republic of China \\ ${ }^{7}$ Guangdong Engineering Technology Center of Nutrition Transformation, Guangzhou 510080, People's Republic of China \\ ${ }^{8}$ Department of Nutrition, School of Public Health, Sun Yat-Sen University, Guangzhou 510080, People's Republic of China \\ ${ }^{9}$ Department of Cardiology, Peking University First Hospital, Beijing 100034, People's Republic of China \\ ${ }^{10}$ Department of Population, Family and Reproductive Health, Johns Hopkins University Bloomberg School of Public Health, \\ Baltimore, MD 21205, USA
}

(Submitted 28 October 2018 - Final revision received 13 April 2019 - Accepted 2 May 2019; First published online 29 July 2019)

\section{Abstract}

We aimed to investigate the association between plasma retinol and incident cancer among Chinese hypertensive adults. We conducted a nested case-control study, including 231 patients with incident cancer and 231 matched controls during a median 4.5-year follow-up of the China Stroke Primary Prevention Trial. There was a significant, inverse association between retinol levels and digestive system cancer (per $10 \mu \mathrm{g} / \mathrm{dl}$ increases: OR $0.79 ; 95 \%$ CI $0.69,0.91)$. When compared with participants in the first quartile of retinol $(<52.3 \mu \mathrm{g} / \mathrm{dl})$, a significantly lower cancer risk was found in participants in quartile $2-4(\geq 52 \cdot 3 \mu \mathrm{g} / \mathrm{dl}$ : OR $0 \cdot 31 ; 95 \%$ CI $0 \cdot 13,0 \cdot 71)$. However, there was a U-shaped association between retinol levels and non-digestive system cancers where the risk of cancers decreased (although not significantly) with each increment of plasma retinol (per $10 \mu \mathrm{g} / \mathrm{dl}$ increases: OR 0.89; $95 \%$ CI $0.60,1.31$ ) in participants with retinol $<68.2 \mu \mathrm{g} / \mathrm{dl}$, and then increased significantly with retinol (per $10 \mu \mathrm{g} / \mathrm{dl}$ increase: OR 1.65; $95 \%$ CI 1.12, 2.44) in participants with retinol $\geq 68.2 \mu \mathrm{g} / \mathrm{dl}$. In conclusion, there was a significant inverse dose-response association between plasma retinol and the risk of digestive system cancers. However, a U-shaped association was observed between plasma retinol and the risk of non-digestive cancers (with a turning point approximately $68 \cdot 2 \mu \mathrm{g} / \mathrm{dl}$ ).

Key words: Retinol: Digestive cancers: Non-digestive cancers: Hypertensive populations

Cancer is a worldwide clinical and public health problem which, in 2013, killed 8.2 million people globally and has become the second leading cause of deaths ${ }^{(1)}$. While the aetiology of cancer is not completely understood, multiple factors including diet, smoking, family history of cancer, ethnicity, genetic factors and nutritional status are postulated to play important roles.

Vitamin A is an essential micronutrient which is derived from dietary sources including animal-based foods or carotenoids from plant-based foods ${ }^{(2,3)}$. Vitamin A plays important roles in cellular growth, differentiation, proliferation and apoptosis ${ }^{(4,5)}$. In animal models, vitamin A may suppress carcinogenesis ${ }^{(4,6)}$. In humans, plasma retinol is the major circulating form of vitamin A and the most commonly used indicator of vitamin A status ${ }^{(7)}$. To date, a number of studies have been conducted to investigate the association between plasma retinol and the incidence of specific forms of cancer, such as gastric ${ }^{(8-11)}$, oesophageal ${ }^{(8)}$, liver ${ }^{(12-14)}$,

Abbreviations: DBP, diastolic blood pressure; RR, relative risk; SBP, systolic blood pressure; tHcy, total homocysteine.

* Corresponding authors: X. Xu, email xipingxu126@126.com or X. Qin, email pharmaqin@126.com or H. Zhang email zhanghaocau@cau.edu.cn 
prostate $^{(15,16)}$ and breast ${ }^{(17-19)}$ cancer, or total cancer ${ }^{(20-23)}$, but found inconsistent results. Overall, most of the previous prospective studies found that high retinol concentrations were significantly associated with decreased risks of $\operatorname{gastric}^{(8,10)}$, oesophageal $^{(8)}$ or liver cancer ${ }^{(12-14)}$. Consistently, Willett et al. ${ }^{(20)}$ also found that although the mean values for retinol were similar for total cancer cases and the matched controls, for gastrointestinal cancer, the retinol levels were substantially lower in cases than their matched controls $(P=0 \cdot 008)$. In contrast, a meta-analysis of eleven nested case-control studies ${ }^{(19)}$ found no significant association between blood retinol and breast cancer. Moreover, a recent meta-analysis ${ }^{(16)}$ of fifteen nested case-control studies found that higher retinol level was associated with increased risk of prostate cancer. Taken together, available studies indicate that the beneficial effect associated with higher retinol concentrations was mainly observed for digestive system cancers. This report aimed to address some remaining gaps on this topic. First of all, few previous studies were specifically focused on investigating the shape of the association between plasma retinol levels and incident cancer risk of digestive system $v$. non-digestive system. Furthermore, few data were available in hypertensive populations, especially in Chinese hypertensive population, who have very different dietary pattern and life style compared with western population. Finally, most previous studies did not fully investigate the modifiers in the association between plasma retinol and cancer risk.

This report analysed the data from the China Stroke Primary Prevention Trial (CSPPT) using a nested cancer case-matched control design. CSPPT showed that the combined use of enalapril and folic acid, compared with enalapril alone, significantly reduced the risk of first stroke among hypertensive adults ${ }^{(24)}$. A greater beneficial effect was seen in those with hypercholesterolemia $^{(25)}$, diabetes ${ }^{(26)}$, higher total homocysteine (tHcy) levels with CC/CT genotypes ${ }^{(27)}$ or lower platelet count ${ }^{(28)}$. Moreover, enalapril-folic acid therapy, compared with enalapril alone, can significantly delay the progression of chronic kidney diseases among hypertensive patients with mild-to-moderate chronic kidney diseases ${ }^{(29)}$, and reduce the mortality risk in hypertensive patients with heavy proteinuria ${ }^{(30)}$, the development of proteinuria in diabetic patients with hypertension ${ }^{(31)}$, and the magnitude of the increase of uric acid concentrations in general hypertensive adults ${ }^{(32)}$. However, enalapril-folic acid therapy had no significant effect on the risk of new-onset diabetes $^{(33)}$ or cancer ${ }^{(34)}$, compared with enalapril alone. In addition, our previous study suggested that elevated tHcy concentrations significantly decreased the antihypertensive effect of the enalapril-based antihypertensive treatment in previously untreated hypertensive patients ${ }^{(35)}$. Furthermore, there was a significant inverse association between plasma retinol and the risk of first stroke among hypertensive adults ${ }^{(36)}$. The present study aimed to examine the relationship of plasma retinol with the incident risk of total cancers and two subtypes: digestive and nondigestive system cancers. Furthermore, this study evaluated possible effect modifiers on the retinol-cancer associations.

\section{Methods \\ Participants}

The study participants for this nested case-control study were drawn from the CSPPT. The methods and major results of the trial have been reported elsewhere ${ }^{(24)}$. Briefly, the CSPPT was a multi-community, randomised, double-blind clinical trial conducted from 19 May 2008 to 24 August 2013 with 20702 hypertensive adults in thirty-two communities in China. Eligible participants were men and women aged $45-75$ years old who had hypertension, defined as seated resting systolic blood pressure (SBP) of $140 \mathrm{mmHg}$ or higher or diastolic blood pressure (DBP) of $90 \mathrm{mmHg}$ or higher at both the screening and recruitment visits, or were taking antihypertensive medication. The major exclusion criteria included history of physician-diagnosed stroke, myocardial infarction, heart failure, coronary revascularisation or congenital heart disease.

A total of 20702 eligible participants were randomly assigned, in a 1:1 ratio, to one of two treatment groups: a daily oral dose of one tablet containing $10 \mathrm{mg}$ enalapril and $0.8 \mathrm{mg}$ folic acid (the enalapril-folic acid group); or a daily oral dose of one tablet containing $10 \mathrm{mg}$ enalapril only (the enalapril group). All participants were followed up every 3 months. At each follow-up visit, vital signs, study drug adherence, concomitant medication use, adverse events and possible endpoint events were documented by trained research staff and physicians. All participants were part of the CSPPT (clinicaltrials.gov identifier: NCT00794885). The CSPPT and the present study were approved by the ethics committee of the Institute of Biomedicine, Anhui Medical University, Hefei, China (FWA assurance number FWA00001263). Written, informed consent was obtained from all participants in the CSPPT.

\section{Ascertainment of endpoints}

Cancer incidence, a pre-specified endpoint of the CSPPT, was the main outcome in this analysis. Cancer was diagnosed based on either positive pathological findings or specific clinical manifestations. Acceptable evidence for pathological findings included original or photocopied pathological reports and original or photocopied medical records from hospitals in which pathological results were cited. When pathological data were not available, cases were independently reviewed by two oncologists. Cancer was diagnosed only when both physicians made the same clinical diagnosis based on clinical manifestations and examinations.

All cancer events were reviewed and adjudicated by an independent Endpoint Adjudication Committee, whose members were unaware of study-group assignments.

\section{Nested case-control study}

During a median treatment duration of 4.5 years, cancer occurred in 116 participants (1.12\%) in the enalapril-folic acid group as compared with 116 participants $(1 \cdot 12 \%)$ in the enalapril group.

Using data from the CSPPT, we conducted a nested casecontrol study of 232 incident cases and 232 matched controls within this cohort. Controls were randomly chosen from the baseline CSPPT participants who did not develop cancer during the follow-up period and were matched for age (no more than 1 year), sex, treatment group and study site with the cases on a 1:1 ratio. After excluding those with missing retinol data, a total of 231 incident cases, which included 129 cases of digestive cancers (53 oesophageal, forty-three gastric, nineteen colorectal and 
fourteen other sites) and 102 cases of non-digestive cancers (twenty-seven breast, twenty-six lung and forty-nine other sites), and 231 matched controls were included in the present analysis (Supplementary Fig. S1).

\section{Laboratory assays}

Overnight fasting venous blood samples of all participants were obtained from each subject at baseline. Plasma retinol was measured by liquid chromatography with tandem quadrupole mass spectrometers (LC-MS/MS) in a commercial lab (Beijing DIAN Medical Laboratory) from August 2016 to July 2017. The interassay CV ranged from $4.66 \%$ to $6.40 \%$, while the intra-assay $\mathrm{CV}$ ranged from $0.54 \%$ to $15.12 \%$. Serum folate was measured by a commercial laboratory using a chemiluminescent immunoassay (New Industrial) from March 2014 to April 2014. Serum tHcy, fasting lipids and glucose levels were measured using automatic clinical analysers (Beckman Coulter) at the core laboratory of the National Clinical Research Center for Kidney Disease, Nanfang Hospital, Guangzhou, China.

\section{Statistical analysis}

We assumed that people with a higher plasma retinol levels represented $25 \%$ of the general population, the estimated OR between the plasma retinol levels and cancer risk was about $0 \cdot 40^{(10,14)}$, the type I error rate was $<0.05(\alpha=0.05)$ and the response rate was $80 \%$. On the basis of these assumptions, 231 cases have at least $90 \%(\beta=0 \cdot 10)$ power to detect the effect size.

Baseline characteristics are presented as means and standard deviations for continuous variables and percentages for categorical variables. Differences in baseline characteristics between cases and controls were compared using conditional logistic regression for categorical variables and generalised paired $t$ tests for continuous variables. OR of total cancers and cancer subtypes were estimated by modelling plasma retinol as a continuous variable (each $10 \mu \mathrm{g} / \mathrm{dl}$ increment) and as quartiles using conditional logistic regression, without and with adjustment for BMI, smoking status, alcohol drinking status, SBP, DBP, total cholesterol, TAG, HDL-cholesterol, fasting glucose, folate and tHcy at baseline, as well as time-averaged SBP and DBP over the trial period. (To convert retinol in $\mu \mathrm{g} / \mathrm{dl}$ to $\mu \mathrm{mol} / \mathrm{l}$, multiply by 0.0349 .) In addition, we applied two-piecewise regression models to examine the threshold effect of plasma retinol levels on total cancers and non-digestive cancers using a smoothing function. The threshold level (turning point) for each was determined using likelihood-ratio tests and bootstrap resampling methods.

In the stratified analysis, possible modifications of the association between plasma retinol as a continuous variable (per $10 \mu \mathrm{g} / \mathrm{dl}$ increment) and the risk of digestive cancers were assessed for variables including age ( $<60 v$. $\geq 60$ years), sex, treatment group (enalapril $v$. enalapril-folic acid), current smoking (no $v$. yes), current alcohol drinking (no $v$. yes), $\operatorname{SBP}(<160$ $v$. $\geq 160 \mathrm{mmHg})$, total cholesterol $(<5.2 v . \geq 5.2 \mathrm{mmol} / \mathrm{l})$ and folate $(<8.6$ (median) $v . \geq 8.6 \mathrm{ng} / \mathrm{ml})$ levels at baseline, and time-averaged SBP $(<140 v . \geq 140 \mathrm{mmHg}$ ) over the trial period.

A two-tailed $P<0.05$ was considered to be statistically significant in all analyses. Analyses were performed using empower software (www.empowerstats.com, X\&Y solutions, Inc.) and R software (http://www.R-project.org/).

\section{Results \\ Characteristics of the participants}

The analysis included 231 cancer cases with complete retinol measurements (Supplementary Fig. S1), which included 129 cases of digestive cancers (fifty-three oesophageal, forty-three gastric, nineteen colorectal and fourteen other sites) and 102 cases of non-digestive cancers (twenty-seven breast, twentysix lung and forty-nine other sites). The distribution of major cancer subtypes is presented in Supplementary Table S1.

Table 1 shows the baseline characteristics of the cases and controls by total cancers, digestive cancers and non-digestive cancers. Mean values of plasma retinol were lower in patients with digestive cancers $(65.8$ (SD 25.9) $\mu \mathrm{g} / \mathrm{dl}$ ) than in control subjects (73.7 (sD 26.4) $\mu \mathrm{g} / \mathrm{dl}$ ); however, values did not differ significantly between cases and control subjects for total cancers and non-digestive cancers.

Cancer cases were more likely to be smokers and had higher time-averaged SBP over the trial period. However, there were no significant differences between cases and controls regarding other variables, including BMI, alcohol drinking, baseline SBP, total cholesterol, TAG, HDL-cholesterol, tHcy and folate, for total cancers or among the two cancer subtypes.

\section{Association of plasma retinol with the risk of total cancers}

Overall, there was a U-shaped association between plasma retinol and total cancers (Fig. 1(a)). For total cancers a turning point of $67.9 \mu \mathrm{g} / \mathrm{dl}$ yielded the best-fitting model in a piecewise regression. The risk of total cancers decreased significantly with each increment of plasma retinol (per $10 \mu \mathrm{g} / \mathrm{dl}$ increases: OR 0.74 ; $95 \%$ CI $0.60,0.92)$ in participants with retinol $<67.9 \mu \mathrm{g} / \mathrm{dl}$, and increased non-significantly with each increment of plasma retinol (per $10 \mu \mathrm{g} / \mathrm{dl}$ increase: OR $1 \cdot 11 ; 95 \% \mathrm{CI} 0 \cdot 94,1 \cdot 30$ ) in participants with retinol $\geq 67.9 \mu \mathrm{g} / \mathrm{dl}$ (Table 2 ).

\section{Association of plasma retinol with the risk of digestive and non-digestive cancers}

The association between plasma retinol and digestive cancers is plotted in Fig. 1(b). There was a significant, inverse association between plasma retinol as a continuous variable and digestive cancers (per $10 \mu \mathrm{g} / \mathrm{dl}$ increases: OR 0.79; $95 \%$ CI 0.69, 0.91). Compared with participants in quartile $1(<52 \cdot 3 \mu \mathrm{g} / \mathrm{dl})$, a significantly lower risk of digestive cancers was found in participants in quartile $2(52.3-<66.4 \mu \mathrm{g} / \mathrm{dl}$; OR 0.39; $95 \% \mathrm{CI} 0 \cdot 16,0.97)$, quartile $3(66 \cdot 4-<83 \cdot 1 \mu \mathrm{g} / \mathrm{dl}$; OR $0 \cdot 25 ; 95 \%$ CI 0.09, 0.67), quartile 4 $\left(\geq 83.1 \mu \mathrm{g} / \mathrm{dl}\right.$; OR $0 \cdot 23 ; 95 \%$ CI 0.08, 0.65; $P_{\text {for trend }}$ for quartiles $1-4=0.006)$ and quartile $2-4(\geq 52 \cdot 3 \mu \mathrm{g} / \mathrm{dl}$; OR $0.31 ; 95 \%$ CI $0 \cdot 13,0 \cdot 71$ ) (Table 3 ). Similar trends were found between plasma retinol and oesophageal cancers (OR 0.58; $95 \%$ CI 0.40, 0.84) or gastric cancers (OR 0.80; $95 \%$ CI 0.62, 1.03) (Supplementary Table S2).

However, there was a U-shaped association between plasma retinol and non-digestive cancers (Fig. 1(c)). For non-digestive cancers a turning point of $68.2 \mu \mathrm{g} / \mathrm{dl}$ yielded the best fitting model in a piecewise regression. The risk of non-digestive 
Table 1. Baseline characteristics among cancer cases and control subjects

(Mean values and standard deviations; numbers of participants and percentages)

\begin{tabular}{|c|c|c|c|c|c|c|c|c|c|c|c|c|c|c|c|}
\hline \multirow[b]{3}{*}{ Characteristics } & \multicolumn{5}{|c|}{ Total cancers } & \multicolumn{5}{|c|}{ Digestive cancers } & \multicolumn{5}{|c|}{ Non-digestive cancers } \\
\hline & \multicolumn{2}{|c|}{$\begin{array}{l}\text { Cases } \\
(n 231)\end{array}$} & \multicolumn{2}{|c|}{$\begin{array}{l}\text { Controls } \\
(n 231)\end{array}$} & \multirow[b]{2}{*}{$P$} & \multicolumn{2}{|c|}{$\begin{array}{l}\text { Cases } \\
(n 129)\end{array}$} & \multicolumn{2}{|c|}{$\begin{array}{l}\text { Controls } \\
(n \text { 129) }\end{array}$} & \multirow[b]{2}{*}{$P$} & \multicolumn{2}{|c|}{$\begin{array}{l}\text { Cases } \\
(n 102)\end{array}$} & \multicolumn{2}{|c|}{$\begin{array}{l}\text { Controls } \\
(n \text { 102) }\end{array}$} & \multirow[b]{2}{*}{$P$} \\
\hline & Mean & SD & Mean & SD & & Mean & SD & Mean & SD & & Mean & SD & Mean & SD & \\
\hline Age (years) & $61 \cdot 8$ & $7 \cdot 0$ & $61 \cdot 8$ & $7 \cdot 0$ & 0.312 & $63 \cdot 6$ & $6 \cdot 5$ & $63 \cdot 6$ & $6 \cdot 4$ & 0.427 & $59 \cdot 6$ & $7 \cdot 2$ & $59 \cdot 6$ & $7 \cdot 2$ & 0.535 \\
\hline Male & & & & & 1.000 & & & & & 1.000 & & & & & 1.000 \\
\hline$n$ & \multicolumn{2}{|c|}{121} & \multicolumn{2}{|c|}{121} & & \multicolumn{2}{|c|}{78} & \multicolumn{2}{|c|}{78} & & \multicolumn{2}{|c|}{43} & \multicolumn{2}{|c|}{43} & \\
\hline$\%$ & \multicolumn{2}{|c|}{$52 \cdot 4$} & \multicolumn{2}{|c|}{$52 \cdot 4$} & & \multicolumn{2}{|c|}{60.5} & \multicolumn{2}{|c|}{$60 \cdot 5$} & & \multicolumn{2}{|c|}{$42 \cdot 2$} & \multicolumn{2}{|c|}{$42 \cdot 2$} & \\
\hline BMl $\left(\mathrm{kg} / \mathrm{m}^{2}\right)$ & $24 \cdot 1$ & 3.6 & 24.4 & $3 \cdot 8$ & 0.365 & 23.3 & 3.4 & 23.9 & 3.8 & 0.142 & $25 \cdot 2$ & 3.5 & $25 \cdot 1$ & 3.6 & 0.720 \\
\hline Current smoking & \multirow{2}{*}{\multicolumn{2}{|c|}{91}} & & & 0.013 & & & & & 0.041 & & & & & 0.150 \\
\hline$n$ & & & 7 & & & 5 & & 4 & & & 3 & & 2 & & \\
\hline$\%$ & 39 & & 30 & & & 44 & & 34 & & & 33 & & 25 & & \\
\hline Current drinking & & & & & 0.900 & & & & & 0.210 & & & & & 0.100 \\
\hline$n$ & 6 & & 6 & & & 4 & & 3 & & & 2( & & 2 & & \\
\hline$\%$ & 28 & & 29 & & & 35 & & 29 & & & 19 & & 28 & & \\
\hline Enalapril-folic acid & & & & & 1.000 & & & & & 1.000 & & & & & 1.000 \\
\hline$n$ & 11 & & 11 & & & 7 & & 7 & & & 4 & & 4 & & \\
\hline$\%$ & 50 & & 50 & & & 55 & & 55 & & & 44 & & 44 & & \\
\hline Blood pressure (mmHg) & & & & & & & & & & & & & & & \\
\hline Baseline SBP & 163.9 & $18 \cdot 8$ & 164.9 & $17 \cdot 3$ & 0.515 & $163 \cdot 3$ & $17 \cdot 6$ & 164.4 & $14 \cdot 4$ & 0.575 & $164 \cdot 6$ & $20 \cdot 2$ & 165.5 & $20 \cdot 4$ & 0.718 \\
\hline Baseline DBP & 92.5 & $11 \cdot 6$ & $93 \cdot 6$ & 11.9 & 0.276 & $92 \cdot 2$ & $11 \cdot 1$ & 91.5 & $12 \cdot 1$ & 0.616 & $92 \cdot 9$ & $12 \cdot 2$ & $96 \cdot 2$ & $11 \cdot 3$ & 0.013 \\
\hline $\begin{array}{l}\text { Time-averaged SBP during } \\
\text { the treatment period }\end{array}$ & 140.5 & $12 \cdot 1$ & $138 \cdot 1$ & $9 \cdot 7$ & 0.018 & $140 \cdot 7$ & $13 \cdot 2$ & 138.8 & 9.3 & 0.178 & $140 \cdot 3$ & $10 \cdot 7$ & $137 \cdot 2$ & $10 \cdot 2$ & 0.035 \\
\hline $\begin{array}{l}\text { Time-averaged DBP } \\
\text { during the treatment period }\end{array}$ & $83 \cdot 1$ & $9 \cdot 0$ & $82 \cdot 2$ & $7 \cdot 8$ & $0 \cdot 198$ & $82 \cdot 7$ & 9.5 & $81 \cdot 0$ & $7 \cdot 5$ & 0.098 & 83.7 & $8 \cdot 3$ & $83 \cdot 7$ & 8.0 & 0.941 \\
\hline Laboratory results & & & & & & & & & & & & & & & \\
\hline Total cholesterol $(\mathrm{mmol} / \mathrm{l})$ & $5 \cdot 4$ & 1.2 & 5.4 & $1 \cdot 2$ & 0.818 & $5 \cdot 2$ & $1 \cdot 1$ & $5 \cdot 3$ & $1 \cdot 3$ & 0.255 & $5 \cdot 6$ & 1.3 & $5 \cdot 4$ & $1 \cdot 1$ & 0.147 \\
\hline HDL-cholesterol (mmol/l) & 1.4 & 0.4 & 1.3 & 0.4 & 0.371 & 1.4 & 0.4 & $1 \cdot 3$ & 0.4 & 0.076 & $1 \cdot 3$ & 0.3 & $1 \cdot 3$ & 0.3 & 0.433 \\
\hline $\mathrm{TAG}(\mathrm{mmol} / \mathrm{l})$ & 1.6 & 0.9 & 1.6 & 0.7 & 0.551 & 1.5 & 0.9 & 1.5 & 0.7 & 0.989 & $1 \cdot 7$ & 1.0 & 1.6 & 0.9 & 0.412 \\
\hline Glucose $(\mathrm{mmol} / \mathrm{l})$ & $5 \cdot 6$ & $1 \cdot 7$ & $5 \cdot 8$ & 1.9 & $0 \cdot 139$ & $5 \cdot 3$ & $1 \cdot 1$ & $5 \cdot 7$ & $2 \cdot 0$ & 0.045 & $5 \cdot 9$ & $2 \cdot 1$ & $5 \cdot 9$ & 1.9 & 0.801 \\
\hline Folate $(\mathrm{ng} / \mathrm{ml})$ & 8.9 & 3.8 & $9 \cdot 1$ & 4.4 & 0.583 & 9.5 & $4 \cdot 1$ & $9 \cdot 7$ & $5 \cdot 0$ & 0.857 & $8 \cdot 1$ & $3 \cdot 2$ & 8.4 & $3 \cdot 3$ & 0.456 \\
\hline Homocysteine $(\mu \mathrm{mol} / \mathrm{l})$ & $14 \cdot 1$ & $5 \cdot 7$ & 14.9 & 8.2 & 0.239 & $14 \cdot 2$ & 4.9 & 14.6 & $6 \cdot 1$ & 0.620 & 14.0 & $6 \cdot 6$ & $15 \cdot 3$ & $10 \cdot 3$ & 0.274 \\
\hline Retinol $(\mu \mathrm{g} / \mathrm{dl})^{*}$ & 68.8 & 26.4 & $71 \cdot 0$ & $25 \cdot 9$ & 0.293 & $65 \cdot 8$ & $25 \cdot 9$ & 73.7 & $26 \cdot 4$ & 0.007 & $72 \cdot 6$ & 26.6 & $67 \cdot 6$ & $25 \cdot 0$ & 0.103 \\
\hline
\end{tabular}

SBP, systolic blood pressure; DBP, diastolic blood pressure.

* To convert retinol in $\mu \mathrm{g} / \mathrm{dl}$ to $\mu \mathrm{mol} / \mathrm{l}$, multiply by 0.0349 .

(a)

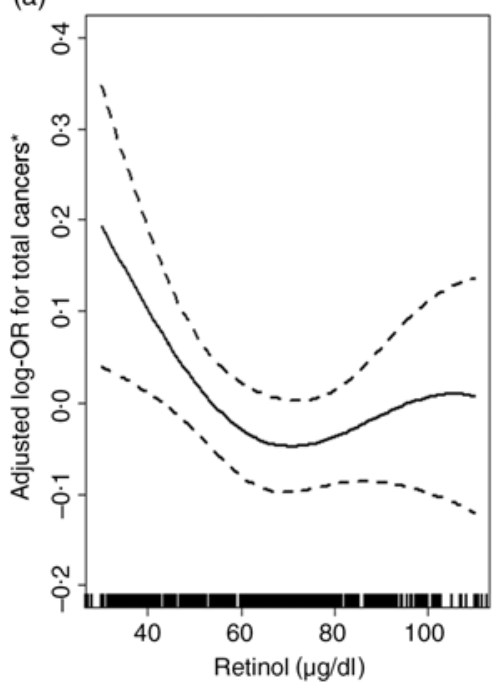

(b)

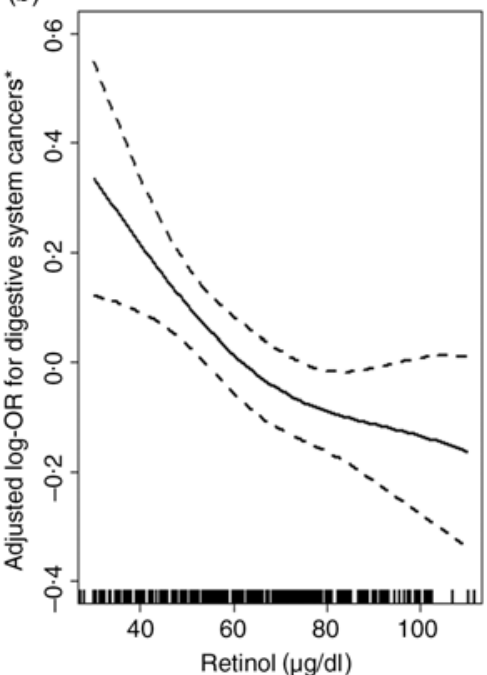

(c)

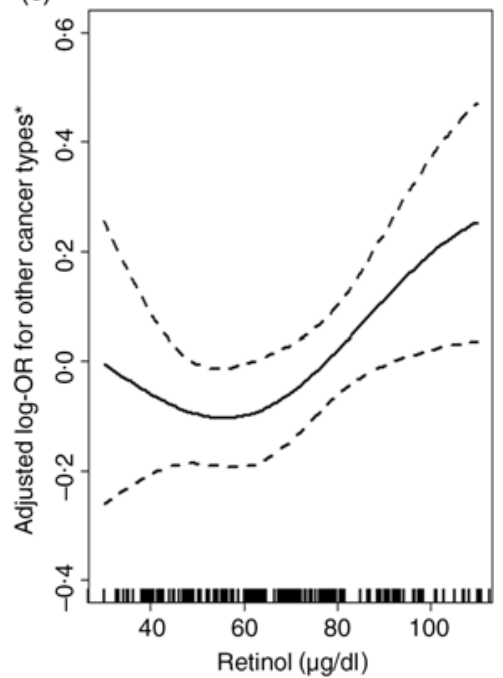

Fig. 1. Relationship of plasma retinol with the risk of total cancers (a), digestive cancers (b) and non-digestive cancers (c). * Adjusted for BMI, smoking status, alcohol drinking status, systolic blood pressure (SBP), diastolic blood pressure (DBP), total cholesterol, TAG, HDL-cholestrol, fasting glucose, folate, total homocysteine at baseline, as well as time-averaged SBP and DBP over the trial period. To convert retinol in $\mu \mathrm{g} / \mathrm{dl}$ to $\mu \mathrm{mol} / \mathrm{l}$, multiply by 0.0349 .

cancers decreased non-significantly with each increment of plasma retinol (per $10 \mu \mathrm{g} / \mathrm{dl}$ increases: OR 0.89; $95 \%$ CI $0.60,1.31)$ in participants with retinol $<68.2 \mu \mathrm{g} / \mathrm{dl}$; and increased significantly with each increment of plasma retinol (per $10 \mu \mathrm{g} / \mathrm{dl}$ increases: OR 1.65; $95 \%$ CI 1.12, 2.44) in participants with retinol $\geq 68.2 \mu \mathrm{g} / \mathrm{dl}$ (Table 2 ). 
Table 2. Threshold effect analyses of retinol levels on the risk of total cancers and non-digestive cancers using two-piecewise regression models

(Odds ratios and $95 \%$ confidence intervals)

\begin{tabular}{|c|c|c|c|c|c|c|c|}
\hline \multirow[b]{2}{*}{ Retinol $(\mu \mathrm{g} / \mathrm{dl}) \dagger$} & \multicolumn{3}{|c|}{ Unadjusted } & \multirow[b]{2}{*}{ Retinol $(\mu \mathrm{g} / \mathrm{dl}) \dagger$} & \multicolumn{3}{|c|}{ Adjusted* } \\
\hline & OR & $95 \% \mathrm{Cl}$ & $P$ & & OR & $95 \% \mathrm{Cl}$ & $P$ \\
\hline \multicolumn{8}{|l|}{ Total cancers } \\
\hline$<67.9$ & 0.80 & $0.66,0.97$ & 0.020 & $<67.9$ & 0.74 & $0.60,0.92$ & 0.007 \\
\hline$\geq 67.9$ & 1.11 & $0.97,1.28$ & 0.130 & $\geq 67.9$ & 1.11 & $0.94,1.30$ & 0.215 \\
\hline \multicolumn{8}{|c|}{ Non-digestive cancers } \\
\hline$<70.8$ & 0.87 & $0.66,1.15$ & 0.334 & $<68.2$ & 0.89 & $0.60,1.31$ & 0.551 \\
\hline$\geq 70.8$ & 1.50 & $1.09,2.06$ & 0.012 & $\geq 68.2$ & 1.65 & $1.12,2.44$ & 0.012 \\
\hline
\end{tabular}

SBP, systolic blood pressure; DBP, diastolic blood pressure.

${ }^{*}$ Adjusted for BMI, smoking status, alcohol drinking status, SBP, DBP, total cholesterol, TAG, HDL-cholesterol, fasting glucose, folate, total homocysteine at baseline, as well as time-averaged SBP and DBP over the trial period.

† To convert retinol in $\mu \mathrm{g} / \mathrm{dl}$ to $\mu \mathrm{mol} / \mathrm{l}$, multiply by 0.0349 .

Table 3. Association between baseline retinol levels (per $10 \mu \mathrm{g} / \mathrm{dl}$ increases) and the risk of digestive system cancers (Odds ratios and $95 \%$ confidence intervals)

\begin{tabular}{|c|c|c|c|c|c|c|c|}
\hline \multirow[b]{2}{*}{ Retinol $(\mu \mathrm{g} / \mathrm{dl}) \dagger$} & \multirow[b]{2}{*}{ Cases/controls } & \multicolumn{3}{|c|}{ Unadjusted } & \multicolumn{3}{|c|}{ Adjusted* } \\
\hline & & OR & $95 \% \mathrm{Cl}$ & $P$ & OR & $95 \% \mathrm{Cl}$ & $P$ \\
\hline Continuous (per-10 $\mu \mathrm{g} / \mathrm{dl}$ increase) & $129 / 129$ & 0.86 & $0.77,0.96$ & 0.009 & 0.79 & $0.69,0.91$ & 0.001 \\
\hline \multicolumn{8}{|l|}{ Quartiles } \\
\hline Q1 $(<52 \cdot 3)$ & $41 / 25$ & 1 & Ref & - & 1 & Ref & - \\
\hline Q2 (52.3-<66.4) & $30 / 32$ & 0.49 & $0.23,1.04$ & 0.062 & 0.39 & $0.16,0.97$ & 0.042 \\
\hline Q3 $(66.4-<83.1)$ & 29/36 & 0.38 & $0.17,0.86$ & 0.020 & 0.25 & $0.09,0.67$ & 0.006 \\
\hline Q4 ( $\geq 83 \cdot 1)$ & 29/36 & 0.37 & $0.16,0.85$ & 0.019 & 0.23 & $0.08,0.65$ & 0.005 \\
\hline$P$ for trend & & & & 0.022 & & & 0.006 \\
\hline Q2-Q4 ( $\geq 52 \cdot 3)$ & $88 / 104$ & 0.43 & $0.22,0.84$ & 0.014 & 0.31 & $0.13,0.71$ & 0.005 \\
\hline
\end{tabular}

SBP, systolic blood pressure; DBP, diastolic blood pressure.

* Adjusted for BMI, smoking status, alcohol drinking status, SBP, DBP, total cholesterol, TAG, HDL-cholesterol, fasting glucose, folate, total homocysteine at baseline, as well as timeaveraged SBP and DBP over the trial period.

$\dagger$ To convert retinol in $\mu \mathrm{g} / \mathrm{dl}$ to $\mu \mathrm{mol} / \mathrm{l}$, multiply by 0.0349 .

To address the possibility of reverse causation, we performed lag analysis and found similar results as we obtained from the conventional analyses as shown in Supplementary Table S3 for digestive system cancers, and Supplementary Table 54 for non-digestive system cancers and total cancers.

\section{Stratified analyses}

Stratified analyses were performed to assess the association between plasma retinol levels (per $10 \mu \mathrm{g} / \mathrm{dl}$ increases) and the risk of digestive cancers (Fig. 2) in various subgroups. None of the variables, including sex, age, treatment group, SBP, current smoking, current alcohol drinking, total cholestrol and folate levels at baseline, as well as SBP over the trial period, significantly modified the protective effects associated with plasma retinol levels ( $P$ for all interactions $>0 \cdot 05$ ).

\section{Discussion}

This nested case-control study demonstrated a significant inverse dose-response association between plasma retinol and the risk of digestive cancers. However, a U-shaped association between retinol and the risk of non-digestive cancers was observed, with a turning point of retinol at $68.2 \mu \mathrm{g} / \mathrm{dl}$. Our findings provide some new information regarding the benefit-risk ratio of vitamin A supplementation and cancer risk.
Our findings are in agreement with the results from several previous prospective studies. Two prospective studies, one in Taiwan (fifty cases) and one in Shanghai (213 cases), both observed that men with higher serum retinol had a reduced risk of liver cancer ${ }^{(13,14)}$. A Finnish prospective cohort study also found that in male smokers, higher serum retinol levels were associated with a lower risk of incident liver cancer (highest quartile $v$. lowest quartile of serum retinol: hazard ratio 0.58 , $95 \%$ CI $\left.0 \cdot 39,0 \cdot 85, P_{\text {for trend }}<0 \cdot 001\right)^{(12)}$. A case-cohort study conducted in Linxian, China, found that serum retinol concentrations were inversely associated with oesophageal cancer risk in male non-smokers (relative risk (RR) $0.79,95 \%$ CI $0 \cdot 63,0 \cdot 99)^{(8)}$. It is worth noting that all of these studies were conducted in males only, or only found the protective effect of retinol on the risk of cancer in males. A European prospective study found an inverse association between plasma retinol and gastric cancer risk in males and females, but did not test sex-specific differences ${ }^{(10)}$. The Linxian study reported that the gastric cardia cancer incidence fell $10 \%$ for each quartile increase of serum retinol (RR 0.90; $95 \%$ CI 0.83, 0.99) $)^{(8)}$. Our results also showed that there was a strong inverse dose-response association between plasma retinol and the risk of digestive cancers. This association was consistent in males and females, as well as smokers and non-smokers.

In contrast to digestive cancers, our results suggested a U-shaped relationship between plasma retinol levels and total 


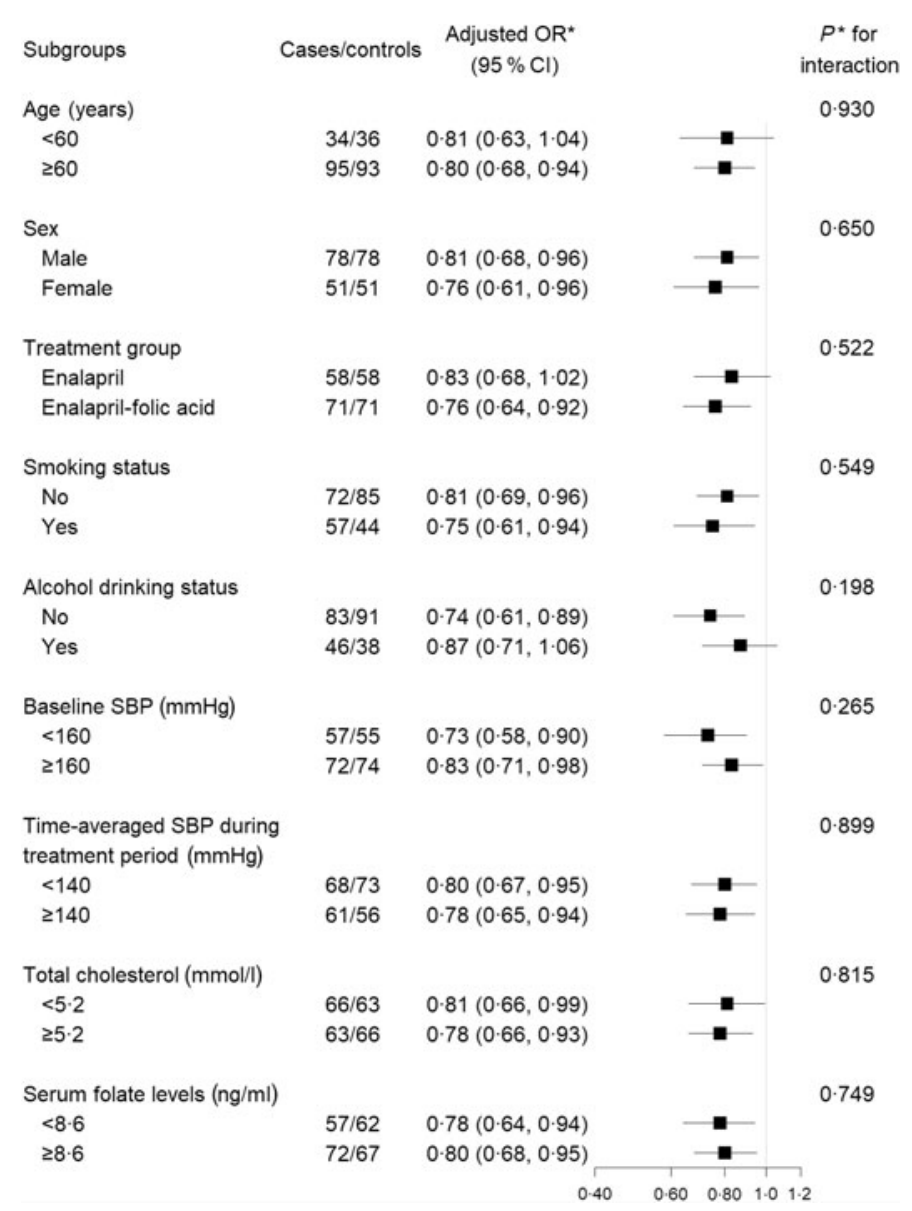

Fig. 2. Stratified analyses of the association between plasma retinol (per $10 \mu \mathrm{g} / \mathrm{dl}$ increment) and the risk of digestive cancers. ${ }^{*}$ Adjusted for BMl, smoking status, alcohol drinking status, systolic blood pressure (SBP), diastolic blood pressure (DBP), total cholesterol, TAG, HDL-cholesterol, fasting glucose, folate, total homocysteine at baseline, as well as time-averaged SBP and DBP over the trial period. To convert retinol in $\mu \mathrm{g} / \mathrm{dl}$ to $\mu \mathrm{mol} / \mathrm{l}$, multiply by 0.0349 .

cancers and non-digestive cancers. The $\beta$-Carotene and Retinol Efficacy Trial (CARET) found that after an average of 4 years of supplementation, the combination of $\beta$-carotene and vitamin A had an adverse effect on the incidence of lung cancer (RR 1.28; $95 \%$ CI 1.04, 1.57), as compared with the placebo group ${ }^{(37)}$. Postintervention follow-up in CARET participants showed a lung cancer RR of 1.12 (95\% CI 0.97, 1.31), suggesting persistent adverse effects but no longer statistically significant ${ }^{(38)}$. Similarly, Peleg et al. observed that the lowest risk of cancer at all sites was found in participants in quintiles three and four of the baseline retinol levels (mean: about $49 \mu \mathrm{g} / \mathrm{dl}$ ) in an Evans County, Georgia study ${ }^{(22)}$. The RR for participants in the second, third, fourth and fifth quintiles were 0.9, 0.5, 0.6 and $1 \cdot 2$, respectively, when compared with those in quintile $1^{(22)}$. The study by Willett et al ${ }^{(20)}$ with a mean retinol value of about $68 \mu \mathrm{g} / \mathrm{dl}(67.3$ and $68.7 \mu \mathrm{g} / \mathrm{dl}$, respectively, for total cancer cases and the matched controls) also indicated a non-significant U-shaped relation of serum retinol with total cancer risk, with the lowest cancer risk in participants in quintiles four of the serum retinol levels. The RR for participants in the second, third, fourth and fifth quintiles were $1 \cdot 0,0 \cdot 9,0 \cdot 7$ and $1 \cdot 1$, respectively, when compared with those in quintile $1^{(20)}$. Furthermore, for lung cancer, the retinol levels were non-significantly higher in cases than their matched controls ${ }^{(20)}$. However, the mean plasma retinol values for cancer cases and the matched controls were only 41 and $36 \mu \mathrm{g} / \mathrm{dl}$, respectively, in the study by Coates et al. ${ }^{(21)}$. Accordingly, when serum retinol was treated as tertiles, the RR for participants in the third tertile were 0.3 for breast cancer, and 0.6 for lung cancer, when compared with those in tertile one ${ }^{(21)}$. In consistent with our results, the above studies also suggest that there may be a U-shaped relationship between plasma retinol levels and non-digestive cancers. Nevertheless, both some of the above studies ${ }^{(20-22)}$ and the present study were severely underpowered for evaluating the association between plasma retinol and specific sites of cancer. Therefore, our findings should be regarded as hypothesis-generating. Future studies with larger sample size, additional time points of retinol assessment and examination of specific forms of cancer are warranted to confirm and expand our work.

The biological mechanism by which retinol affects cancer risk is not clear. Retinol has been thought to play a role in reducing the risk of cancers by affecting the regulation of cell growth, differentiation and apoptosis ${ }^{(4-6)}$. Furthermore, retinol has direct anti-inflammatory and antioxidant activities ${ }^{(39)}$. As the gastrointestinal tract is thought to be a major site of antioxidant action ${ }^{(40)}$, retinol may therefore be especially protective 
against digestive cancers. The possible mechanisms for the increased risk of non-digestive cancers associated with higher retinol levels are still uncertain. However, it has been suggested that vitamin A may be a double-edged sword. Both a deficiency and an excess of retinol have similar adverse effects in terms of fibrosis and carcinogenesis ${ }^{(41)}$. Large doses of vitamin A are associated with embryonic malformations and can cause damage to the skin, bone mineral density, nervous system and internal organs ${ }^{(42-46)}$. Consistently, our study found that the risk of non-digestive cancers increased with each increment of retinol in those participants with plasma retinol $\geq 68.2 \mu \mathrm{g} / \mathrm{dl}$. However, more studies are needed to confirm our results and to further investigate the biological mechanisms underlying the associations.

Our study had several strengths. First, the nested case-control design in the context of a large prospective population (20 702 hypertensive adults) reduced possibility of selection bias. Second, we revealed different effects of plasma retinol on different types of cancer, providing new insights in this field. However, our study also had some limitations. First, plasma retinol levels of the study participants were only assessed at baseline. Therefore, we could not evaluate the effect of plasma retinol changing on the incidence of cancer. Second, we did not have the detailed dietary information about the sources of vitamin A. Previous studies suggested that Chinese adults derive vitamin A mainly from plant food sources ${ }^{(47)}$. Whether our findings could be extrapolated to populations with animal foods as the major sources of retinol should be further examined in future studies. Third, the participants of the present study were all hypertensive Chinese patients; whether our findings can be extrapolated to non-Chinese or populations without hypertension remain to be investigated. For example, for non-digestive cancers, compared with controls, cases had significantly lower DBP levels at baseline and higher time-averaged SBP levels during the treatment period. However, adjustment for baseline blood pressure and time-averaged blood pressure during the treatment period did not substantially change the findings. Fourth, although major potential confounders were controlled for, it is likely that the results may have been affected by unmeasured or unidentified confounders. Lastly, our study had a relatively small sample size. Although we have stratified the analysis by two subtypes, non-digestive and digestive cancers, the number of site-specific cancers was small; thus, this study was severely underpowered for evaluating the association of plasma retinol levels with specific sites of cancer. Therefore, our study was just hypothesisgenerating, and all findings need to be further investigated and confirmed in future studies.

In conclusion, our study found a significant inverse doseresponse association between plasma retinol levels and the risk of digestive system cancers. However, a U-shaped association was found for non-digestive cancers with plasma retinol turning point of $68.2 \mu \mathrm{g} / \mathrm{dl}$.

\section{Acknowledgements}

This work was supported by the National Key Research and Development Program (2018ZX09301034003, 2016YFE0205400,
2018ZX09739), the Science and Technology Planning Project of Guangzhou, China (201707020010); the Science, Technology and Innovation Committee of Shenzhen (KQCX20120816105958775, JSGG20170412155639040, GJHS20170314114526143, KC2014JSCX0071A); President Foundation of Nanfang Hospital, Southern Medical University (2017C007); and Outstanding Youths Development Scheme of Nanfang Hospital, Southern Medical University (2017J009).

X. X., X. Q., H. Z. and X. W. contributed to research idea and study design. L. X., Y. S., T. L., H. G., B. W., G. T., Y. Z., J. L., Y. H., X. W., H. Z. and X. Q. were involved in data acquisition. L. X., Y. S., T. L. and X. Q. were involved in data analysis and interpretation. L. X. and C. L. performed statistical analysis. L. X., Y. S., T. L., H. G., B. W., G. T., C. L., W. H., Y. Y., W. L., Y. Z., J. L., Y. H., X. W., H. Z., X. Q. and X. X. carried out review and revision of the article.

X. X. reports grants from the Science and Technology Planning Project of Guangzhou, China (201707020010) and the Science, Technology and Innovation Committee of Shenzhen (KQCX20120816105958775, JSGG20170412155639040, GJHS20170314114526143, KC2014JSCX0071A). X. Q. reports grants from the President Foundation (2017C007) and Outstanding Youths Development Scheme of Nanfang Hospital, Southern Medical University (2017J009). B. W. reports grants from the National Key Research and Development Program (2016YFE0205400, 2018ZX09739, 2018ZX09301034003). No other disclosures were reported.

\section{Supplementary material}

To view supplementary material for this article, please visit https://doi.org/10.1017/S000711451900120X

\section{References}

1. GBD 2013 Mortality and Causes of Death Collaborators (2015) Global, regional, and national age-sex specific all-cause and cause-specific mortality for 240 causes of death, 1990-2013: a systematic analysis for the Global Burden of Disease Study 2013. Lancet 385, 117-171.

2. Krinsky NI \& Johnson EJ (2005) Carotenoid actions and their relation to health and disease. Mol Aspects Med 26, 459-516.

3. Harrison EH (2012) Mechanisms involved in the intestinal absorption of dietary vitamin A and provitamin A carotenoids. Biochim Biophys Acta Mol Cell Biol Lipids 1821, 70-77.

4. Sporn MB (1983) Role of retinoids in differentiation and carcinogenesis. Cancer Res 43, 3034-3040.

5. Gudas LJ \& Wagner JA (2011) Retinoids regulate stem cell differentiation. J Cell Physiol 226, 322-330.

6. Altucci L \& Gronemeyer H (2001) The promise of retinoids to fight against cancer. Nat Rev Cancer 1, 181-193.

7. Edem DO (2009) Vitamin A: a review. Asia Pac J Clin Nutr 1, 65-82.

8. Abnet CC, Qiao YL, Dawsey SM, et al. (2003) Prospective study of serum retinol, beta-carotene, beta-cryptoxanthin, and lutein/ zeaxanthin and esophageal and gastric cancers in China. Cancer Causes Control 14, 645-655.

9. Yuan JM, Ross RK, Gao YT, et al. (2004) Prediagnostic levels of serum micronutrients in relation to risk of gastric cancer in Shanghai, China. Cancer Epidemiol Biomarkers Prev 13, 1772-1780. 
10. Jenab M, Riboli E, Ferrari P, et al. (2006) Plasma and dietary carotenoid, retinol and tocopherol levels and the risk of gastric adenocarcinomas in the European prospective investigation into cancer and nutrition. Br J Cancer 95, 406-415.

11. Persson C, Sasazuki S, Inoue M, et al. (2008) Plasma levels of carotenoids, retinol and tocopherol and the risk of gastric cancer in Japan: a nested case-control study. Carcinogenesis 29, 1042-1048.

12. Lai GY, Weinstein SJ, Albanes D, et al. (2014) Association of serum alpha-tocopherol, beta-carotene, and retinol with liver cancer incidence and chronic liver disease mortality. $\mathrm{Br}$ J Cancer 111, 2163-2171.

13. Yu MW, Hsieh HH, Pan WH, et al. (1995) Vegetable consumption, serum retinol level, and risk of hepatocellular carcinoma. Cancer Res 55, 1301-51305.

14. Yuan JM, Gao YT, Ong CN, et al. (2006) Prediagnostic level of serum retinol in relation to reduced risk of hepatocellular carcinoma. J Natl Cancer Inst 98, 482-490.

15. Mondul AM, Watters JL, Mannisto S, et al. (2011) Serum retinol and risk of prostate cancer. Am J Epidemiol 173, 813-821.

16. Key TJ, Appleby PN, Travis RC, et al. (2015) Carotenoids, retinol, tocopherols, and prostate cancer risk: pooled analysis of 15 studies. Am J Clin Nutr 102, 1142-1157.

17. Maillard V, Kuriki K, Lefebvre B, et al. (2010) Serum carotenoid, tocopherol and retinol concentrations and breast cancer risk in the E3N-EPIC study. Int J Cancer 127, 1188-1196.

18. Pouchieu C, Galan P, Ducros V, et al. (2014) Plasma carotenoids and retinol and overall and breast cancer risk: a nested case-control study. Nutr Cancer 66, 980-988.

19. Hu F, Wu Z, Li G, et al. (2015) The plasma level of retinol, vitamins $\mathrm{a}, \mathrm{C}$ and $\alpha$-tocopherol could reduce breast cancer risk? A meta-analysis and meta-regression. J Cancer Res Clin 141, 601-614.

20. Willett WC, Polk BF, Underwood BA, et al. (1984) Relation of serum vitamins A and $\mathrm{E}$ and carotenoids to the risk of cancer. $N$ Engl J Med 310, 430-434.

21. Coates RJ, Weiss NS, Daling JR, et al. (1988) Serum levels of selenium and retinol and the subsequent risk of cancer. $\mathrm{Am}$ $J$ Epidemiol 128, 515-523.

22. Peleg I, Heyden S, Knowles M, et al. (1984) Serum retinol and risk of subsequent cancer: extension of the Evans County, Georgia, study. J Natl Cancer Inst 73, 1455-1458.

23. Knekt P, Aromaa A, Maatela J, et al. (1990) Serum vitamin A and subsequent risk of cancer: cancer incidence follow-up of the Finnish Mobile Clinic Health Examination Survey. Am J Epidemiol 132, 857-870.

24. Huo Y, Li J, Qin X, et al. (2015) Efficacy of folic acid therapy in primary prevention of stroke among adults with hypertension in China: the CSPPT randomized clinical trial. JAMA 313, $1325-1335$.

25. Qin X, Li J, Spence JD, et al. (2016) Folic acid therapy reduces the first stroke risk associated with hypercholesterolemia among hypertensive patients. Stroke 47, 2805-2812.

26. Xu RB, Kong X, Xu BP, et al. (2017) Longitudinal association between fasting blood glucose concentrations and first stroke in hypertensive adults in China: effect of folic acid intervention. Am J Clin Nutr 105, 564-570.

27. Zhao M, Wang X, He M, et al. (2017) Homocysteine and stroke risk: modifying effect of Methylenetetrahydrofolate Reductase C677T Polymorphism and Folic Acid Intervention. Stroke 48, 1183-1190.
28. Kong X, Huang X, Zhao M, et al. (2018) Platelet count affects efficacy of folic acid in preventing first stroke. $\mathrm{J} \mathrm{Am}$ Coll Cardiol 71, 2136-2146.

29. Xu X, Qin X, Li Y, et al. (2016) Efficacy of folic acid therapy on the progression of chronic kidney disease. JAMA Intern Med 176, 1443.

30. Li Y, Qin X, Luo L, et al. (2017) Folic acid therapy reduces the risk of mortality associated with heavy proteinuria among hypertensive patients. J Hypertens 35, 1302-1309.

31. Li Y, Liang M, Wang G, et al. (2017) Effects of folic acid therapy on the New-Onset proteinuria in Chinese hypertensive patients. Hypertension 70, 300-306.

32. Qin X, Li Y, He M, et al. (2017) Folic acid therapy reduces serum uric acid in hypertensive patients: a substudy of the China Stroke Primary Prevention Trial (CSPPT). Am J Clin Nutr 105, 882-889.

33. Qin X, LiJ, Zhang Y, et al. (2016) Effect of folic acid supplementation on risk of new-onset diabetes in adults with hypertension in China: findings from the China Stroke Primary Prevention Trial (CSPPT). J Diabetes 8, 286-294.

34. Qin X, Shen L, Zhang R, et al. (2017) Effect of folic acid supplementation on cancer risk among adults with hypertension in China: a randomized clinical trial. Int J Cancer 141, 837-847.

35. Qin X, Li Y, Sun N, et al. (2017) Elevated homocysteine concentrations decrease the antihypertensive effect of AngiotensinConverting enzyme inhibitors in hypertensive patients. Arterioscler Thromb Vasc Biol 37, 166-172.

36. Yu Y, Zhang H, Song Y, et al. (2019) Plasma retinol and the risk of first stroke in hypertensive adults: a nested case-control study. Am J Clin Nutr 109, 449-456.

37. Omenn GS, Goodman GE, Thornquist MD, et al. (1996) Effects of a combination of beta carotene and vitamin A on lung cancer and cardiovascular disease. N Engl J Med 334, 1150-1155.

38. Goodman GE, Thornquist MD, Balmes J, et al. (2004) The BetaCarotene and Retinol Efficacy Trial: incidence of lung cancer and cardiovascular disease mortality during 6-year follow-up after stopping beta-carotene and retinol supplements. $J$ Natl Cancer Inst 96, 1743-1750.

39. Palace VP, Khaper N, Qin Q, et al. (1999) Antioxidant potentials of vitamin A and carotenoids and their relevance to heart disease. Free Radic Biol Med 26, 746-761.

40. Halliwell B, Zhao K \& Whiteman M (2000) The gastrointestinal tract: a major site of antioxidant action? Free Radic Res $\mathbf{3 3}$, 819-830.

41. Leo MA \&Lieber CS (1999) Alcohol, vitamin A, and betacarotene: adverse interactions, including hepatotoxicity and carcinogenicity. Am J Clin Nutr 69, 1071-1085.

42. Rothman KJ, Moore LL, Singer MR, et al. (1995) Teratogenicity of high vitamin A intake. N Engl J Med 333, 1369-1373.

43. Melhus H, Michaelsson K, Kindmark A, et al. (1998) Excessive dietary intake of vitamin A is associated with reduced bone mineral density and increased risk for hip fracture. Ann Intern Med 129, 770-778.

44. Colbert MC (2002) Retinoids and cardiovascular developmental defects. Cardiovasc Toxicol 2, 25-39.

45. Biesalski HK (1989) Comparative assessment of the toxicology of vitamin A and retinoids in man. Toxicology 57, 117-161.

46. Hathcock JN, Hattan DG, Jenkins MY, et al. (1990) Evaluation of vitamin A toxicity. Am J Clin Nutr 52, 183-202.

47. Du W, Wang H, Wang Z, et al. (2018) Dietary vitamin an intake among Chinese adults: findings from CNTCS2015. NutrJ 17, 60. 